\title{
Déterminants de la performance des Institutions de Microfinance en Côte d'Ivoire : Une approche de la méthode DEA en deux étapes
}

\author{
Dr Eric Kokola KOUADIO ${ }^{1}$, Dr Vadoua BAMBA ${ }^{2}$ \\ ${ }^{I}$ Université Jean Lorougnon Guédé de Daloa, UFR SEG, Côte d'Ivoire, Daloa \\ ${ }^{2}$ Université Jean Lorougnon Guédé de Daloa, UFR SEG, Côte d'Ivoire, Daloa
}

\begin{abstract}
Résumé : L'objectif de cet article est d'identifier les déterminants de la performance sociale, financière et globale des institutions de microfinance (IMF) en Côte d'Ivoire sur la période 2013-2018. Apres avoir estimé les scores d'efficacité à partir de la méthode « Data Envelopment Analysis » (méthode DEA) dans la première étape, nous avons régressé ces scores sur une série de variables explicatives dans la deuxième étape à partir modèle Tobit. La méthode de bootstrap a été également utilisée pour la robustesse de l'étude. Les données sont issues de la période 2013-2018, et collectées à la direction de la microfinance en Côte d'Ivoire. L'échantillon concerne 21 IMF sur un total de 42 ayant transmis leur rapport d'activité au cours de la même période. Les résultats des estimations montrent que la rentabilité des actifs (ROA), les IMF nouvelles, les IMF de types coopératifs et l'année d'existence (l'âge) sont significatifs et exercent une influence négative sur les performances financière, sociale et globale. En effet, les IMF de petites tailles sont significatives à $1 \%$ et influencent la performance financière, tandis que la rentabilité des actifs (ROA) et les IMF de types coopératifs influencent la performance sociale respectivement à $5 \%$ et $10 \%$. Enfin, l'année d'existence (l'âge) exerce une influence significative à $10 \%$ sur la performance globale.
\end{abstract}

Mots clefs : IMF, performance des IMF, méthode DEA, Méthode bootstrap et modèle Tobit.

\section{Introduction}

L'exclusion financière des pauvres du système financier formel classique est un problème de développement social qui, aujourd'hui retient l'attention des dirigeants partout dans le monde. Selon Demirguc-Kunt et Klapper (cité dans Togba, 2019), en Afrique subsaharienne, l'exclusion financière touche environ $76 \%$ des personnes adultes, taux au-dessus de la moyenne globale de $50 \%$ et celui des économies à revenus élevés qui est de $11 \%$. Les gouvernements, les donateurs et les institutions financières internationales reconnaissent que l'accès aux services financiers peut jouer un rôle important dans la réduction de la pauvreté et la vulnérabilité des personnes pauvres. 
Dans l'espace de l'Union Economique et Monétaire Ouest Africaine (UEMOA), la microfinance est un secteur en pleine expansion et porteur de beaucoup d'espoir sur le plan de l'accessibilité aux services financiers pour les populations de la zone.

Concernant le cas de la Côte d'Ivoire, suite à la crise armée de septembre 2002 et les crises postélectorales de 2010-2011, les institutions de microfinance ivoiriennes ont été fragilisées. Le secteur a été particulièrement perturbé. Les indicateurs restent mitigés, c'est le cas des encours de crédits qui ont subi des évolutions contrastées en passant de 18,98\% à 3,38\% entre 2007 et 2009 (CNM, 2010). Le contexte de crise post-électorale a engendré une hausse de la pauvreté (46,3\% en 2015), on note une croissance des indicateurs de volume d'activité (INS/DGPLP, 2015) et une dégradation importante de la qualité du portefeuille de crédits en 2018, qui s'établit à 13,4\% (DGTCP-DRSSFD, 2020). Ces pertes ont induit un déficit important, après deux (2) années de résultats positifs (2016 et 2017). En dépit de cette situation, les perspectives restent favorables pour le secteur de la microfinance, car la confiance des clients demeure intacte, au regard de l'accroissement de leur nombre. La restructuration en cours de l'UNACOOPEC-CI et la poursuite des actions d'assainissement devraient avoir un impact positif sur le secteur.

La performance des institutions de microfinance (IMF) en Côte d'Ivoire à sortir les pauvres du seuil de pauvreté est désormais posée. En effet, la dégradation des indicateurs financiers peut entrainer la cessation des activités et affecter la performance sociale, ce qui pourrait engendrer des problèmes au plan économique et social du fait de leur taille dans le système de financement décentralisé. A contrario, une bonne performance des IMF entrainerait des perspectives de croissance et de développement, qui permettront à la fois l'atteinte des objectifs financiers (amélioration des taux de remboursement, de la qualité du portefeuille de crédit, etc.) et sociaux (amélioration du niveau de vie, hausse du pouvoir d'achat, etc.).

Pour des organisations internationales telles que la Banque Mondiale ou les Nations Unies, il faut parvenir à la construction d'un secteur de microcrédit solide afin de mettre en place des organisations de microfinance pérennes touchant un maximum de populations pauvres. Elles préconisent dans ce sens, l'institutionnalisation des programmes de microfinance, notamment la mise en place d'organisation de microcrédits rentables, répondant aux lois des marchés (De Briey, 2005). Il faut noter que les IMF sont des organisations hybrides qui combinent des objectifs financiers et sociaux (Battilana \& Dorado, 2010). Par conséquent, évaluer la performance des IMF par le seul prisme d'indicateurs financiers (la rentabilité) s'avère peu pertinent. Comme le suggère Balkenhol (2007), l'analyse se révèle plus fructueuse en l'abordant en termes d'efficacité. Une IMF qui utilise efficacement les ressources dont elle dispose pourrait améliorer simultanément la qualité de son portefeuille ainsi que son résultat.

Dans la littérature, la performance des IMF est généralement considérée comme une fonction de déterminants internes et externes. Les déterminants internes proviennent des comptes bancaires (bilans 
et / ou des comptes de profits et de pertes) et pourraient donc être appelés micro ou déterminants spécifiques de la banque. Les déterminants externes sont des facteurs qui ne sont pas liés à la gestion des IMF, mais reflètent la situation économique et l'environnement juridique (Benzai, 2016). Ceux-ci affectent le fonctionnement et la performance des institutions financières. Charnes et Cooper, (1980) sont les premiers à évoquer la nature spécifique des facteurs non contrôlables. Ils reflètent les restrictions organisationnelles et managériales qui affectent la performance des observations et qui l'influencent favorablement ou défavorablement. Pour Gonzalez (2007), Hudon et Balkenhol, (2011), les principaux facteurs explicatifs de la performance des IMF sont la structure de propriété, la méthodologie de prêt, l'âge et la zone d'opération.

C'est donc, dans cette démarche que s'inscrit notre travail, qui vise à identifier les facteurs explicatifs de la performance sociale, financière et globale des IMF en Côte d'Ivoire sur la période 20132018. Nous cherchons, d'abord, à obtenir des scores d'efficacité sociale, financière et globale sur un échantillon de 21 IMF issues de la période 2013-2018, à partir de la méthode Data Envelopment Analysis (DEA). Ensuite, nous régressons les scores obtenus, à partir du modèle Tobit sur une série de facteurs explicatifs, après avoir recouru à la méthode Bootstrap pour la robustesse de l'étude.

L'étude présente plusieurs intérêts: Au plan économique, nous estimons que les décideurs doivent mettre l'accent sur des séances la formation régulières et le respect de la règlementation pour parvenir à une microfinance solide et performante afin de contribuer à la lutte contre la pauvreté. Au niveau théorique, l'étude vise à mettre en exergue les pensées welfaristes et institutionnalistes dans le champs de la microfinance en Côte d'Ivoire. Enfin, sur le plan empirique, nous identifions à partir des estimations des facteurs susceptibles d'influencer les niveaux de performances des IMF. Ces facteurs peuvent constituer une entrave au développement du secteur et par conséquent remettre en cause la mission première qui est la réduction de la pauvreté (performance sociale).

L'étude est organisée en trois (03) parties: La revue de littérature empirique, l'approche méthodologique et les résultats et discussions.

\section{Revue de littérature empirique}

La notion de performance dans cette étude fait allusion au concept d'efficacité qui relève de la façon dont une IMF réussit à allouer ses ressources (actifs, peel et subventions) pour produire un maximum de résultats (nombre de prêts, autosuffisance financière ou impact sur la pauvreté). Les facteurs explicatifs de la performance des IMF ont fait l'objet dersonn nombreux travaux. De manière générale, ces travaux présentent des résultats divergents de l'influence de ces facteurs sur les performances sociale, financière et globale des IMF.

Qayyum et Ahmad (2006) ont évalué l'efficacité de 85 IMF du Sud d'Asie. Les résultats révèlent que l'inefficacité des IMF de certains pays est principalement de nature technique. Pour rendre 
performante ces institutions, elles sont amenées à rehausser les compétences directoriales et améliorer la technologie. Soulama (2008) mesure l'efficacité des institutions de microfinance au Burkina Faso par la méthode DEA (Data Envelopment Analysis) sur un échantillon de 94 coopératives d'épargne et de crédit observé sur l'année 2005. L'auteur montre que l'amélioration de l'efficacité des IMF passe par une amélioration de l'efficacité technique, liée à l'insuffisance de formation, d'équipement et de la qualité de la gestion.

De son côté, Sufian, (2006) analyse l'efficacité des institutions financières non bancaires (IFNB) de la Malaisie en application de la méthode non paramétrique DEA. L'étude révèle que la taille et la part du marché ont un effet négatif sur l'efficacité. Il conclut que les institutions financières non bancaires (IFNB) les plus efficaces tendent à être plus profitables. Dans la même veine, Bassem (2008), en utilisant la même méthode (méthode DEA) pour mesurer l'efficacité de 35 IMF de la région méditerranéenne au cours de la période 2004-2005, montre que les institutions de microfinance de tailles moyennes présentent une plus grande efficacité technique. Ces résultats sont similaires à Kablan (2012) qui montre que les institutions de grande taille sont moins efficaces que celles de petite taille. Selon l'auteure, plus la taille d'une institution augmente, plus les coûts de contrôle et de gestion ne deviennent très élevés.

Varman (2008) de son coté, analyse l'efficacité de 26 institutions de microfinance en Inde en utilisant la méthode des frontières stochastiques. Même si, cette méthode paramétrique est plus précise, le choix des inputs et outputs reste cohérent avec ceux d'une institution financière. Son étude permet de montrer que les institutions financières non bancaires (IFNB) sont plus efficaces que les organisations non gouvernementales. Haq et al. (2010) vont dans le même sens. En évaluant la performance des IMF sur la base des types d'IMF, ils montrent que les institutions de microfinance non gouvernementales sont plus efficaces que les institutions de microfinance filiale de banques commerciales dans la poursuite de réduction de la pauvreté et la soutenabilité financière. Cette conclusion est tout de même critiquable dans la mesure où les inputs choisis sont le travail, le coût par emprunteur, le coût par épargnant et les deux outputs sont les épargnants et les emprunteurs par agent de microfinance. Ceux-ci ne donnent aucune information sur le rôle des IMF dans la réduction de la pauvreté. Servin et al. (2012) montrent que les IMF à but non lucratif affichent un degré d'efficacité technique plus faible que les institutions commerciales, alors que les ONG sont plus efficaces socialement (Gutiérrez et al., 2009; Wijesiri et al., 2015).

Cornée et Thenet (2016) montrent que le statut d'ONG agit positivement sur l'efficacité sociale, tandis que la méthode du prêt individuel et l'âge agissent négativement. En revanche, l'influence de ces trois facteurs sur l'efficacité financière n'est pas significative. Alors que Caudill et al. (2009) ; Nghiem et al. (2006); Wijesiri et al. (2015) montrent que l'effet de l'âge sur la performance financière est positif grâce à des gains d'expérience. Par contre, Hermes et al. (2011) montrent que cet effet s'avère négatif 
et ils justifient leur résultat par le fait que les IMF les plus récentes intègrent la connaissance déjà existante dans le secteur bâtissant ainsi une technologie de production qui leur permet de surclasser les autres organisations. Hartarska et Mersland (2012) trouvent quant à eux, que cet effet est neutre. L'étude de Wijesiri et al. (2015) montre une relation négative entre l'âge et l'efficacité sociale, tandis que Gutiérrez et al. (2009) rapportent une corrélation positive mais non significative. Djontu (2019) montre que la taille du conseil d'administration a un impact positif sur la performance des institutions de microfinance.

Pour Hudon et Balkenhol, (2011), le contexte dans lequel évoluent les IMF est également susceptible d'influencer leur efficacité. Si les IMF se sont initialement développées dans les zones rurales, comme par exemple en Amérique Latine (Morduch, 1999a), elles se répandent aujourd'hui plus rapidement en milieu urbain où elles se révèlent plus performantes (Hulme \& Moore, 2007 ; Vanroose, 2008).

Enfin, Morvant-Roux (2011) et Périlleux, (2013) montrent que les techniques de prêt auxquelles font recourent les IMF, sont plus adaptées aux activités économiques des zones urbaines qu'au secteur agricole, ce qui freine le développement d'institutions socialement et financièrement efficaces. Par contre, Nghiem et al. (2006) notent sans réellement parvenir à expliquer leur résultat contre-intuitif, que l'efficacité financière s'améliore à mesure que l'on s'éloigne des centres urbains. Brown (2005) mentionne que les facteurs qui impactent la performance des IMF peuvent être le nombre de clients servis, le taux de remboursement, les coûts d'administration et les salaires, les taux d'intérêt sur les dépôts et les prêts. Ces facteurs reliés directement à l'institution. L'auteur met l'accent sur l'importance d'atteindre un niveau d'économie d'échelle optimal, car c'est la façon la plus efficace pour aller chercher davantage de clients dans la perspective d'avoir le meilleur impact possible sur la lutte contre la pauvreté. Ces facteurs peuvent aussi être «externes», à l'instar de la qualité des infrastructures à disposition des IMF (Gonzalez, 2007).

Bref, nous pouvons constater que de nombreux travaux ont mis l'accent sur les facteurs d'influence de la performance sociale, financière et globale des IMF dans les régions du monde. Cependant, rares sont les travaux qui se sont intéressés aux facteurs explicatifs dans le cas de la Côte d'Ivoire. C'est pourquoi, il nous parait opportun de réfléchir sur les facteurs d'influences de la performance des IMF en Côte d'Ivoire. Nous souhaitons que l'identification de ces facteurs puisse permettre à relever le défi du développement du secteur, favoriser une plus grande accessibilité de la population exclue du système bancaire et contribuer enfin à la réduction de la pauvreté.

La question est de savoir, quelle approche méthodologique adoptée pour une analyse de ces facteurs en Côte d'Ivoire?

\section{Approche méthodologique}

Pour l'estimation des niveaux d'efficacité des IMF, nous optons pour la méthode DEA (Data Envelopment Analysis). En effet, l'efficacité des IMF a été étudiée à de nombreuses reprises, à l'aide de cette méthode. Á l'instar de Coelli et al. (1998), Cornée \& Thenet (2016), Kobou et al. (2010), nous 
allons recourir à la méthode Data Envelopement Analysis (DEA) pour évaluer les niveaux d'efficacité des IMF en Côte d'Ivoire. Cette méthode offre une analyse basée sur l'évaluation relative de l'efficacité dans une situation input/output multiple, en prenant en compte chaque IMF et en mesurant sa performance relative à une surface d'enveloppement composée des meilleures IMF. Elle génère des niveaux d'efficacité à partir des informations fournies sur les inputs et les outputs, ce qui permet de distinguer les IMF ayant les meilleures pratiques dans le secteur. Cette meilleure pratique est définie comme la frontière de possibilités de production dans l'espace des outputs et inputs. La frontière révèle le niveau maximum de production accessible pour une utilisation donnée des inputs ou de façon équivalente, la quantité minimale d'inputs nécessaire pour produire un niveau d'output donné. Dans cette optique, une IMF est efficace, si par rapport à l'output obtenu, et aux inputs consommés, elle se situe sur la frontière. L'avantage de cette méthode est qu'elle n'impose pas de forme fonctionnelle à la fonction de coût ou de production et permet une combinaison hétérogène des inputs et outputs.

L'approche utilisée consiste à spécifier d'abord les modèles DEA et les variables inputs/outputs, et ensuite, mettre l'accent sur les déterminants de la performance.

\subsection{Spécification des modèles DEA et des variables inputs/outputs \\ 3.1.1 Spécification des modèles DEA}

La méthode DEA est composée de deux modèles : le modèle à rendement d'échelle variable (VRS) et le modèle à rendement d'échelle constant (CRS). Le premier modèle s'applique lorsque toutes les organisations ont atteint leur taille optimale, c'est-à-dire, évoluent dans un environnement de concurrence parfaite, ce qui est rarement le cas. A partir de ce modèle, on calcule un score d'efficacité appelé Constant Returns to Scale Technical Efficiency (CRSTE). Le deuxième modèle est privilégié dans les cas de concurrence imparfaite ou de marchés régulés. A partir de ce modèle VRS, on calcule un score d'efficacité appelé variable return to scale technical efficiency (VRSTE).

Pour spécifier notre modèle, nous recourons à l'approche de production à l'instar de (Kablan, 2012 ; Nzongang, 2011 ; Nzongang et al., 2012; Thenet, 2006, etc.). Selon Kablan, (2012), cette approche nous permet d'intégrer des produits autres que financiers et qui ont un caractère social.

Le programme mathématique linéaire utilisé pour calculer les degrés d'efficacité sous l'hypothèse de rendement d'échelle constant (CRS) est de la forme :

$$
\begin{aligned}
& \operatorname{Min}_{k}-\varepsilon \sum_{r=1}^{s} s_{r}-\varepsilon \sum_{i=1}^{m} s_{i} \\
& s C \\
& y_{r k}-\sum_{j=1}^{n} \lambda_{j} y_{r j}+s_{r}=0 \quad r=1, \ldots, s \\
& \theta_{k} x_{i k}-\sum_{j=1}^{n} \lambda_{j} x_{i j}-s_{i}=0 \quad i=1, \ldots, m \\
& \lambda_{j}, s_{r} \text { et } s_{j} \geq 0 \quad \forall j=1, \ldots, n \quad r=1, \ldots, s \quad i=1, \ldots, m
\end{aligned}
$$

Avec, 
$S_{r}$ : Les outputs en deficit pour chaque output r ou slacks d'outputs ;

$S_{i}$ : Les inputs en exces pour chaque input i ou slacks d'inputs ;

$\varepsilon$ : Une valeur non archimédienne se définissant comme une valeur plus petite que n'importe quel nombre réel positif. $\varepsilon$ est supérieur à 0 .

Le programme mathématique linéaire utilisé pour calculer les degrés d'efficacité sous l'hypothèse de rendement d'échelle variable (VRS) est le suivant :

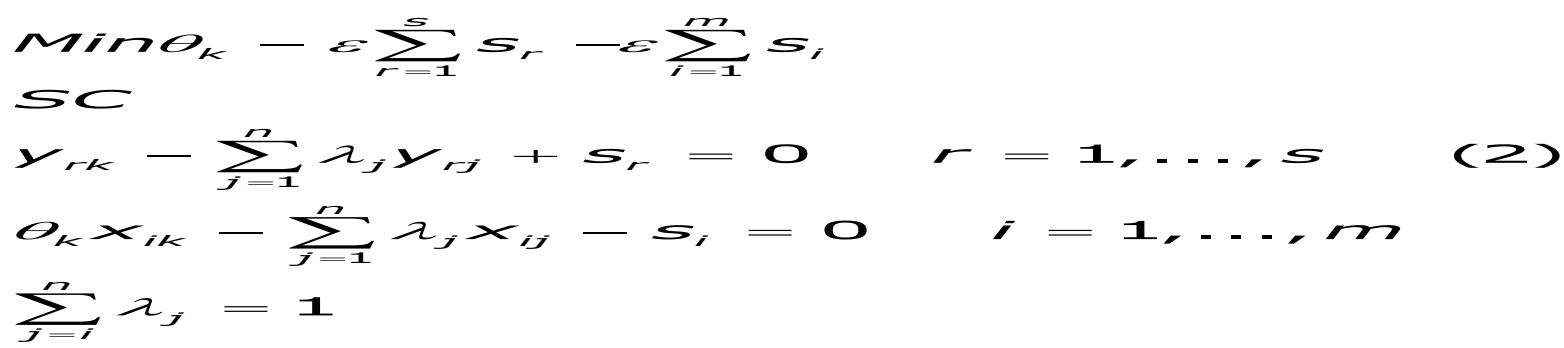

La résolution des programmes CRS et VRS permet d'obtenir des scores d'efficacité technique des IMF étudiées. Cependant, ne sachant pas si les conditions permettant un modèle à rendements constants sont complètement respectées, nous utilisons systématiquement les deux types de rendements (constants et variables) à des fins de robustesse.

Dans la pratique, deux approches de programmation linéaire sont le plus souvent utilisées pour résoudre le problème : l'approche orientée vers les outputs où la somme pondérée des outputs est maximisée tout en maintenant constants les inputs et l'approche orientée vers les inputs ${ }^{1}$, la somme pondérée des inputs est minimisée en maintenant constant les outputs. Comme on pouvait le constater dans son objectif premier, les IMF maximisent leur production en faveur des plus pauvres, à partir d'un panier de ressources donné. Ainsi, une orientation outputs pourrait être privilégiée. Toutefois, comme le soutient (Huguenin, 2013), l'orientation du modèle doit être fonction des inputs et outputs que les décideurs exercent le plus grand pouvoir de gestion. Cornée et Thenet, (2016) soulignent que les gestionnaires en microfinance, contrôlent plus facilement les inputs.

Nous privilégions l'orientation «input » car nous pensons qu'elle serait la mieux adaptée dans cette étude.

\subsubsection{Spécification des variables inputs et outputs}

Pour spécifier les inputs et outputs, nous nous inspirons de plusieurs travaux (Benston, 1965 ; Betl \& Murphy, 1968 ; Berg et al., 1992 ; Cornée \& Thenet, 2016; Kablan, 2012 ; Nzongang, 2011 ; Nzongang et al., 2012; Thenet, 2006, Sealey \& Lindlay, 1977 ; Yaron, 1994) ; etc.).

${ }^{1}$ Les expressions « orientation input » et « orientation output » sont dérivées des équations duales de chacun des modèles, op.cit. Huguenin, (2013) 
Selon Yaron (1994), la performance des IMF est analysée à travers la viabilité financière et la portée sociale. La viabilité renvoie à l'autosuffisance financière, autrement dit, à la capacité de l'institution à générer des revenus financiers grâce à son activité de prêt. La portée sociale correspond à la capacité d'un programme de microfinance à servir le maximum d'emprunteurs.

Les variables « revenu financier » ou «produits d'exploitation » mesurent la viabilité, tandis que, « nombre d'emprunteurs », «nombre de prêts », «encours de crédit» ou encore «nombre de groupes » correspondent à la portée. Lorsque l'étude cherche à mesurer plus la profondeur qui est une dimension de la portée du programme, c'est-à-dire, la capacité de l'institution à servir les emprunteurs les plus pauvres ou les plus fragiles, les variables, «nombre de femmes emprunteuses» et, moins fréquemment, «nombre d'emprunteurs pauvres» sont incluses. Ainsi, nous mesurons la performance sociale, financière et globale des IMF en Côte d'Ivoire en spécifiant un modèle comprenant les variables inputs et outputs.

- Les variables « inputs » sont les suivantes : «les fonds propres », «les charges financières », «les charges du personnel $»$ et «le total actif».

- Les « outputs» sont fonction des types de performance. On distingue les « outputs de performance sociale» et les « outputs de performance financière». Pour le niveau de performance sociale, les variables outputs comprennent «le nombre de prêt encours», "le solde moyen de prêt par emprunteur », «le nombre d'adhérent » et «le pourcentage de femmes emprunteuses ». Au niveau de la performance financière, nous utilisons les variables outputs composées de "la Rentabilité des Actifs_ROA», «l'Autosuffisance Opérationnelle_AO» et «le ratio de capitalisation ».

- Le modèle de performance globale, comprend tous les inputs et outputs de la performance sociale et financière. Le tableau ${ }^{\circ} 1$ résume quelques statistiques des inputs et outputs utilisés.

Tableau 1: Synthèse des variables inputs et outputs retenues

\begin{tabular}{|l|l|l|l|l|}
\hline Variables & Définitions & Moy & Min & Max. \\
\hline$C H P(I)$ & Charges de personnel & 21158.5 & 2.0 & 2234338.0 \\
\hline$A C T(I)$ & Total actifs & 11677.5 & 5.3 & 104988.0 \\
\hline$F P(I)$ & Fonds propres & 1219.12 & -31984.00 & 137717.00 \\
\hline CHF $(\mathrm{I})$ & Charges financières & 360.943 & 0.020 & 10977.62 \\
\hline PRETENC $(\mathrm{O}-\mathrm{PS})$ & Nombre de prêt encours & 13738.3 & 3.0 & 525560.0 \\
\hline Adhérents $(\mathrm{O}-\mathrm{PS})$ & Nombre d'adhérents & 51154 & 152 & 1000541 \\
\hline SMEMP $(\mathrm{O}-\mathrm{PS})$ & Solde moyen de prêt par emprunteur & 946861 & 1155 & 5932302 \\
\hline$P F E(O-P S)$ & Pourcentage de femmes emprunteuses & 40.25 & 14.41 & 96.91 \\
\hline$R O A(O-P F)$ & $\begin{array}{l}\text { Rentabilité des Actifs } \\
\text { = résultat net/total actif }\end{array}$ & -5.450 & -132.210 & 68.190 \\
\hline$A O(O-P F)$ & Autosuffisance Opération. & 101.29 & 10.56 & 493.97 \\
\hline$C A P(O-P F)$ & Ratio de capitalisation & -15.32 & -645.57 & 107.77 \\
\hline
\end{tabular}

(I) : Inputs ; (O-PS) : Outputs de la Performance sociale ; (O-PF) : Output de la Performance financière.

Source : Auteurs

Afin de déterminer les niveaux d'efficacité des IMF en Côte d'Ivoire, nous avons utilisé le logiciel $\mathrm{R}$ version 4.0.2. Les résultats sont présentés dans le tableau $n^{\circ} 2$. 
Tableau 2 : Méthode DEA, résultats des niveaux de performance des IMF en Côte d'Ivoire

\begin{tabular}{|l|l|l|l|l|l|}
\hline Type de modèle & Type de Performance & $\begin{array}{l}\text { Scores } \\
\text { d'efficacité }\end{array}$ & Min. & Max. & $\begin{array}{l}\text { \% IMF } \\
\text { efficaces }\end{array}$ \\
\hline $\begin{array}{l}\text { Modèle à rendement } \\
\text { d'échelle variable } \\
\text { (VRS) }\end{array}$ & Performance Sociale & 0.684 & 0.09591 & 1.00000 & 37,30 \\
\cline { 2 - 7 } & Performance Financière & 0.463 & 0.0005444 & 1.00000 & 30,95 \\
\cline { 2 - 7 } & Performance Globale & 0.824 & 0.1420 & 1.00000 & 57,14 \\
\hline $\begin{array}{l}\text { Modèle à rendement } \\
\text { d'échelle contant } \\
\text { (CRS) }\end{array}$ & Performance Sociale & 0.533 & 0.03349 & 1.00000 & 24,60 \\
\cline { 2 - 7 } & Performance Financière & 0.362 & 0.0005042 & 1.00000 & 22,22 \\
\cline { 2 - 7 } & Performance Globale & 0.557 & 0.01674 & 1.000000 & 23,01 \\
\hline
\end{tabular}

Source : Auteurs

Cette première étape a permis de déterminer les scores d'efficacité des IMF. Dans la deuxième étape, nous mettons l'accent sur l'identification des variables susceptibles d'influencer les niveaux de performance.

\subsection{Analyse des déterminants de la performance des Institutions de Microfinance 3.2.1 Cadre d'analyse}

Dans le domaine de la microfinance, l'approche la plus couramment employée pour identifier les déterminants de la performance des IMF consiste à effectuer dans une première étape l'estimation des scores d'efficacité à partir des variables discrétionnaires (inputs et outputs) et dans une seconde étape, à régresser les scores d'efficacité sur une série de variables explicatives. Les variables incluses en deuxième étape sont des variables exogènes parce que provenant de l'environnement. La question est alors de savoir, si l'inefficacité est due à une mauvaise utilisation des ressources ou à des circonstances extérieures en dehors des IMF?

Les méthodes en deux étapes se concentrent sur l'influence qu'exerce l'environnement sur les scores d'efficacité. À l'aide d'une régression où la distribution de la variable dépendante est censurée (la plupart du temps à l'aide d'un modèle Tobit), on tente d'expliquer les variations de la mesure d'efficacité dans l'ensemble des organisations analysées par une série de variables indépendantes (Simar \& Wilson, 2007). En général, cela suppose que les variables expliquant l'inefficacité sont celles que le gestionnaire ne contrôle pas dans le processus de production. Cette hypothèse est introduite pour éviter le biais inclus dans la première étape, selon lequel le niveau d'inefficacité est indépendant de ces variables alors que dans la deuxième étape, ils sont considérés comme dépendants. L'avantage de cette méthode est qu'en cas d'erreur de spécification dans la deuxième étape, le biais affecte uniquement les coefficients estimés des déterminants et non les coefficients de la frontière. La régression effectuée lors de la deuxième étape peut suivre la méthode des moindres carrés ordinaires (MCO) ou le modèle Tobit pour tenir compte du caractère tronqué (entre 0 et 1) de la variable dépendante (efficacité).

Des études récentes telles que Cornée et al. (2016), Djimasra (2009), Kablan (2012), Kendo (2014), Kobou et al. (2010), Sika (2011), etc., montrent que le modèle Tobit censuré est plus approprié pour identifier les déterminants de l'efficacité des organisations. Selon Kobou et al. (2010), le niveau d'efficacité d'une IMF prend des valeurs dans l'intervalle] 0 1], on ne peut donc pas estimer cette 
équation par les moindres carrés ordinaires (MCO), mais par des modèles censurés tels que le modèle de poisson généralisé et censuré ou le modèle Tobit censuré. Les modèles de régression censurés sont particulièrement recommandés lorsque les données utilisées sont censurées, en d'autres termes, les valeurs de la variable endogène appartiennent à un intervalle précis. Le modèle de poisson est souvent conseillé lorsque les valeurs de la variable dépendante sont des entiers naturels. Alors que, le modèle Tobit est utilisé quand deux conditions sont réunies à savoir : la variable dépendante est continue dans un intervalle et la probabilité pour que la variable dépendante prenne des valeurs nulles est positive.

Pour le cas des déterminants de l'efficacité des IMF, la variable dépendante « le niveau d'efficacité» est continue dans l'intervalle] 0 1]. Le modèle Tobit censuré n'est pas approprié car cette variable n'admet pas des valeurs nulles (Greene, 1995 ; Maddala, 1983) ${ }^{2}$. De même, le modèle de poisson généralisé ne peut pas être utilisé car les valeurs de la variable dépendante ne sont pas des entiers naturels. Pour contourner cette difficulté, les auteurs expliquent l'inefficacité des IMF, en ayant recours au modèle Tobit censuré, puisque le niveau d'inefficacité des IMF prend des valeurs nulles et positives et est continu dans l'intervalle [0 1[. La variable dépendante sera censurée en gardant dans l'échantillon les observations pour lesquelles la valeur de la variable dépendante est nulle comme dans le cas d'espèce où le niveau d'inefficacité des IMF prend des valeurs dans l'intervalle [0 1[.

Xue et Harker (1999) ont mis en exergue un problème plus fondamental relatif à la dépendance statistique des scores d'efficacité. Selon ces auteurs, le fait d'enlever une unité décisionnelle de l'échantillon modifie par construction le score des autres unités décisionnelles. Le fait que les scores DEA obtenus soient relatifs et non pas absolus violent une des hypothèses basiques du modèle de régression, à savoir l'indépendance des individus au sein de l'échantillon. La solution proposée par Xue et al. (1999) consiste à estimer les coefficients de la régression Tobit par la méthode du bootstrap. Simar et Wilson (2007) suggèrent que 2000 itérations soient suffisantes pour obtenir des intervalles de confiance fiables pour les paramètres de la régression. Selon N'Gbo (1991), la méthode de bootstrap fournit un cadre d'analyse de la stabilité des résultats face à des variations d'échantillons. Elle a été utilisée dans les modèles économétriques habituels par des auteurs comme (Freedman, 1981; Rayner, $1990)^{3}$. La forme du modèle Tobit de l'efficacité technique peut s'écrire comme suit :

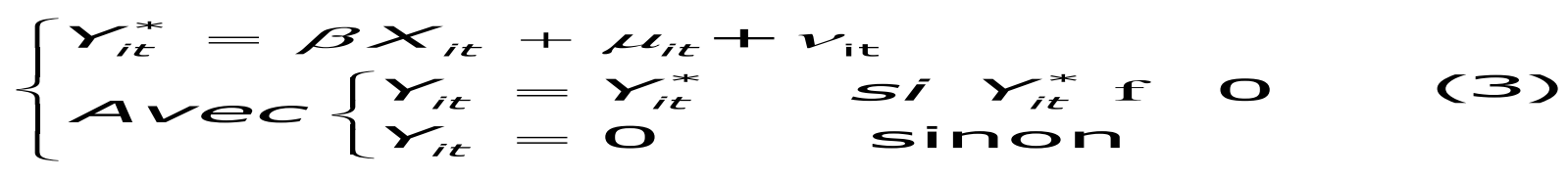

Dans ce travail, le niveau d'efficacité en termes de performance sociale, financière et globale est la variable dépendante $\left(Y_{i t}\right)$. Celle-ci est continue dans l'intervalle] 01 1].

Cependant, si l'on admet avec Greene, (1995) et Madalla, (1993) que l'utilisation du Tobit censuré est appropriée lorsque la variable dépendante prend les valeurs nulles, il convient alors de redéfinir cette dernière variable (cité par Ndjokou, 2013). Ainsi, l'analyse est faite sur la base l'inefficacité (Ineff). La nouvelle variable dépendante ainsi définie prend les valeurs nulles et est continue dans l'intervalle $[0,1[$.

\footnotetext{
${ }^{2}$ op. cit. Kobou et al. (2010)

${ }^{3} \mathrm{Op}$. cit. N'Gbo
} 


\subsubsection{Données de l'étude et variables du modèle $>$ Données de l'étude}

Les données utilisées dans le cadre de cette étude sont issues de la base de données de la Direction de la Microfinance (DM) en Côte d'Ivoire. Cette structure reçoit les états financiers de l'ensemble des IMF en Côte d'Ivoire et établit des rapports financiers trimestriels, semestriels et annuels. L'échantillon concerne vingt une (21) IMF sur un total de cinquante (50) IMF ayant déposé régulièrement leurs états financiers à la Direction de la Microfinance, au 31 décembre 2018.

\section{$>$ Analyse des variables du modèle}

Au regard de la littérature, nous pensons que les variables relatives aux spécificités des IMF, à leurs technologies de distribution de produits ainsi que, les variables relatives à l'environnement socioéconomique dans lequel évoluent les IMF, devraient avoir un impact sur les performances de ces institutions. Notre analyse porte sur les variables suivantes :

\section{- La dette dans l'actif total (dette)}

La part des dettes dans l'actif total de l'IMF correspond au rapport entre l'endettement total et le total actif. Généralement, les IMF avec un grand portefeuille d'emprunt sur le total des actifs pourraient se développer rapidement. Cependant, ce portefeuille peut ne pas être bien diversifié et donc plus exposé au risque de crédit, étant donné que l'expertise en gestion des risques de crédit reste limitée. Cette situation peut entraîner des coûts plus élevés (ou la perte de revenus) en termes de prêts improductifs, ce qui pourrait avoir un effet négatif sur l'efficacité. Néanmoins, ces IMF peuvent apparaître plus rentables, si un risque de crédit élevé se traduit par la hausse des marges d'intérêt. Selon Fama (1990), un taux d'endettement élevé peut être synonyme de confiance parce qu'il justifie la solidité des activités de l'institution, d'où une large proportion des dettes dans la structure de financement. Myer et Majluf, (1984), prônent l'autofinancement pour maintenir la valeur de l'entreprise. La « dette » montre comment les IMF gèrent leur portefeuille de prêt. Plus ce ratio est élevé, plus les IMF prennent des risques importants relativement à la garantie que représente leurs fonds propres et donc moins sera importante leur efficacité. La dette devrait avoir un effet négatif sur la performance financière des IMF.

\section{- La rentabilité des actifs ou Return on Assets (ROA),}

La rentabilité des actifs (ROA) renseigne sur la performance de gestion des actifs de l'IMF visant à optimiser leur rentabilité. Elle mesure la façon dont l'institution utilise ses actifs. C'est une mesure générale de rentabilité qui reflète aussi bien la marge de profit que l'efficacité de l'institution. Les actifs productifs se composent généralement des dépôts à vue, de tous les autres dépôts rémunérés, de l'encours brut de crédits et des placements à long terme. Ils peuvent être considérés comme les éléments sur lesquels les dirigeants peuvent agir dans le cadre de leurs efforts pour maximiser la rentabilité. Les IMF qui tirent le maximum de revenus de leurs actifs seraient aussi les plus pérennes (Mersland \& Strom, 2009). Ce qui laisse entendre que les IMF les plus rentables sont aussi les plus efficaces financièrement et socialement. Hermes et Lensink (cité dans Solhi \& Rigard, 2014) corroborent cet argument en 
affirmant que les IMF les plus rentables contribueraient plus à l'atteinte de l'objectif social se rapportant à la réduction de la pauvreté.

\section{- La forme juridique (forme),}

Le cadre juridique régissant le secteur de la microfinance en Côte d'Ivoire définit deux formes juridiques des institutions de microfinance : les institutions coopératives ou mutualistes (COOP) et les institutions non mutualistes ou sociétés anonymes de microfinance (SA). Selon Akella et Greenbaum, (1988), les coopératives sont caractérisées par leur petite taille, leur permettant de proposer des services financiers (épargne et crédit) et non financiers à des cibles homogènes. Ainsi, comme le soulignent Adair et Berguiga, (2010), les coopératives ou les mutuelles au regard de leur statut peuvent avoir la capacité d'atteindre les plus pauvres. Contrairement aux IMF de type société anonyme (SA) qui placeraient de l'importance sur la rentabilité financière (Morduch 2000 ; Adair \& Berguiga, 2010 ; Gutierrez-Nieto et al., 2007; Mersland \& Strom, 2009; Lafourcade et al., 2005; Hassan \& Sanchez, 2009). Ces institutions sélectionnent leur clientèle en ciblant leurs meilleurs clients auxquels elles accordent des financements plus élevés. Ainsi, nous pensons que les IMF de type coopératif sont susceptibles d'atteindre la performance sociale alors que les IMF de type société anonyme pourraient avoir un impact significatif sur la performante financière.

- L'année d'existence, (âge)

L'âge, représenté par l'année d'existence des IMF est un indicateur important dans la performance. Il peut affecter positivement l'efficacité des IMF, par l'effet d'apprentissage. La relation entre l'âge des IMF et l'efficacité financière et sociale est ancrée dans la théorie des effets d'expérience (Cornée \& Thenet, 2016 ; Wijesiri et al., 2015). Pour Gonzalez (2007), les IMF les plus anciennes ont des ratios de charges opérationnelles plus faibles que les IMF nouvelles. Ceci est justifié en partie par le fait que les anciennes IMF ont fini par maîtriser les pratiques de gestion par un processus d'essais et d'erreurs au fil du temps (Hermes \& Lensink, 2011; Nghiem et al., 2006). Le nombre d'années d'existence confère de l'expertise à l'IMF et lui permet de remplir rapidement et efficacement ses prérogatives (Kablan, 2012; Adair \& Berduiga, 2010). En effet, ces institutions ont probablement constitué des réserves au fil du temps, ce qui leur permettrait de s'engager dans le financement des clients plus pauvres et d'élargir ainsi leur portée sans nuire à leur efficience financière. Ainsi, plus une IMF mûrit, plus elle acquiert de l'expérience, mieux elle parvient à gérer ses coûts et à mettre en place de meilleurs mécanismes de gestion du risque et plus elle atteindra ses objectifs. Par conséquent, l'âge peut affecter positivement l'efficacité globale des IMF par l'effet d'apprentissage.

\section{- La taille des IMF (taille)}

La relation entre la taille des IMF et l'efficacité est analysée à la lumière de la théorie des économies d'échelle (Gonzalez, 2007). Selon Kablan (2012), la taille peut conduire à des économies d'échelle dans la distribution des produits financiers. Ainsi, les IMF qui sont capables d'augmenter leur portée peuvent devenir financièrement rentables. Hansmann (1996) associe la taille et l'intérêt commun des coopératives à leur efficacité. Elles seraient moins efficaces à mesure que leur taille augmente et que l'intérêt commun devient plus diffus. En effet, en grandissant, les coopératives perdent leur avantage informationnel sur les associés car la proximité sociale, culturelle et/ou géographique est moindre. 
Akhavein (1997) et Smirlock (1985) trouvent une relation positive et significative entre la taille et la rentabilité des banques (Benzai, 2016).

\section{- La zone d'implantation (zone)}

La zone d'implantation des IMF permet de capter les niveaux de performances due à la différence des degrés de professionnalisation que l'on observe dans les diverses régions. L'environnement socioéconomique dans lequel évoluent les IMF peut avoir un impact sur leur efficacité. En Côte d'Ivoire, (56,7\%) des IMF sont concentrées au sud du pays avec des points de services contre 34,62\% pour le seul district d'Abidjan. Une faible représentation enregistrée dans les autres régions du pays $(17,2 \%$ au centre, 10,8\% au nord, au 10,7\% à l'est et 4,8\% à l'ouest), (DGTCP, 2018). Au regard de ces chiffres, nous retenons comme zone d'implantation « la zone Abidjan » et la «zone hors Abidjan ». La modalité « zone Abidjan », prend la valeur 1 si l'IMF est implantée à Abidjan et 0 , sinon. Nous attendons une performance financière élevée en «zone Abidjan» et une performance globale moindre «hors Abidjan ».

Le tableau ci-dessous présente une description statistique des variables du modèle.

Tableau 3 : Statistique descriptive des variables explicatives du modèle

\begin{tabular}{|c|c|c|c|c|}
\hline Variables & Définitions & Min. & Moy. & Max. \\
\hline dette & La part des dettes dans l'actif total & 0.2300 & 0.9235 & 4.6800 \\
\hline ROA & La rentabilité des actifs & -132.210 & -5.450 & 68.190 \\
\hline forme & $\begin{array}{l}\text { La forme juridique des IMF comprend deux modalités : } \\
\text { 1. si l'IMF est de type coopératif } \\
\text { 2. si l'IMF est une Société Anonyme }\end{array}$ & \multicolumn{3}{|c|}{ Variables qualitatives } \\
\hline âge & $\begin{array}{l}\text { L'année d'existence des IMF, comprend } \\
\text { trois modalités: } \\
\text { 1. si l'IMF est « nouvelle», elle a moins de } 5 \text { années d'existence } \\
\text { 2. si l'IMF est « jeune ». Elle est comprise entre } 5 \text { ans et } 8 \text { ans } \\
\text { 3. si l'IMF est « mature », elle a plus de } 8 \text { ans. }\end{array}$ & 1.000 & 8.357 & 21.000 \\
\hline taille & $\begin{array}{l}\text { La taille de l'IMF, mesurée par le montant de l'encours de crédit. Elle } \\
\text { est composée de trois modalités : } \\
\text { 1. si l'IMF est de petite taille (Encours de crédit < } 1000000000 \\
\text { Fcfa). } \\
\text { 2. si l'IMF est de taille moyenne (Encours de crédit compris entre } \\
4000000000 \text { et } 100000000000 \text { Fcfa). } \\
\text { 3. Si l'IMF est de grande taille (Encours de crédits > } 4000000000 \\
\text { Fcfa d'encours de crédit) }\end{array}$ & 0.57 & 8027.21 & 100954.0 \\
\hline zone & $\begin{array}{l}\text { Zone d'implantation, une variable binaire, elle prend les modalités: } \\
1 . \text { si l'IMF est implantée Abidjan, } \\
0 . \text { sinon, }\end{array}$ & \multicolumn{3}{|c|}{ Variables qualitatives } \\
\hline
\end{tabular}

Source : Auteurs

Apres la spécification des variables explicatives, le modèle empirique peut ainsi s'écrire :

Ineff $_{i t}=\alpha+\beta_{1} \operatorname{det} t e_{i t}+\beta_{2}$ ROE $_{i t}+\beta_{3}$ forme $_{i t}+\beta_{4}$ taille $_{i t}+\beta_{5} \hat{a ̂ g e}_{i t}+\beta_{6}$ zone $_{i t}+\varepsilon_{i t}$

Avec : 
Ineff $f_{i t}$ représente la variable dépendante qui exprime les niveaux d'inefficacité de chaque IMF obtenus à la date $\mathrm{t}$ en faisant le calcul (1-efficacité des IMF); les scores d'efficacité ont été générés lors de l'estimation du modèle DEA; $\alpha$ est le coefficient constant; $\beta_{1}, \beta_{2}, \beta_{3}, \beta_{4}, \beta_{5}$ et $\beta_{6}$ sont respectivement les coefficients des variables «dette », «ROE», «forme », «taille», «âge», et «zone »; $\varepsilon_{i}$ correspond aux résidus ; i représentent le nombre d'observation (21 IMF) ; t, représente la période d'étude (2013 - 2018).

\section{Résultats et discussion}

\subsection{Présentation des résultats}

L'estimation des déterminants de l'inefficacité des IMF est réalisée grâce au logiciel R, version 4.0.2. La méthode boostrap a été utilisée avec 2000 itérations (nombre de réplique). Les résultats présentés dans le tableau n ${ }^{\circ} 4$ montrent que le modèle Tobit-bootstrap est globalement significatif à $1 \%$ et la p-value $<5 \%$. Les trois colonnes des performances (performance financière, sociale, et globale) répliquent les estimations du modèle VRS.

Tableau 4: Estimation des déterminants de la performance des IMF à partir du modèle bootstrap-Tobit sur les scores d'inefficacité.

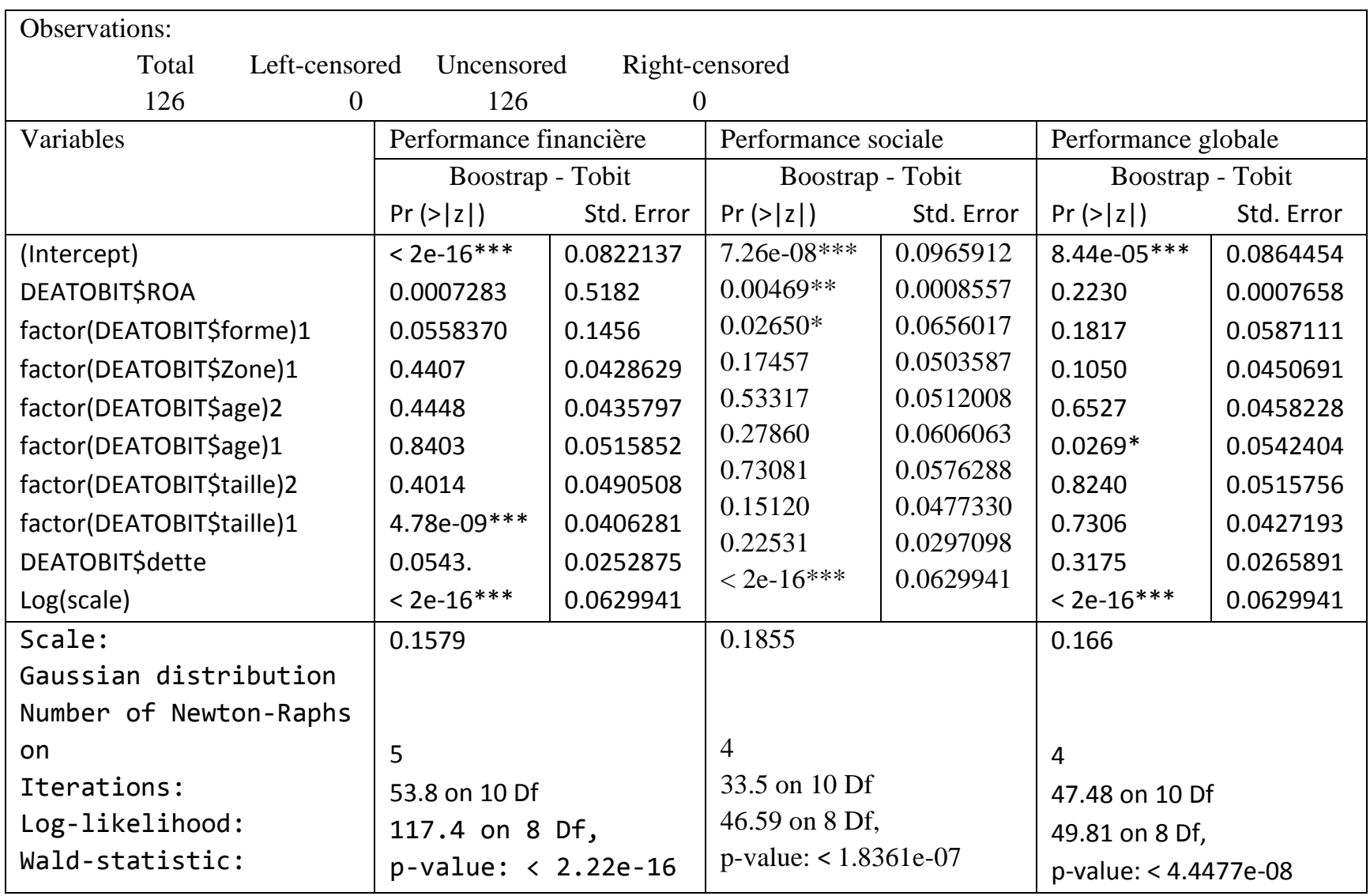

***Significatif à $1 \% ; \quad * *$ Significatif à $5 \% ; \quad *$ Significatif à $10 \%$

Source : Auteur, à partir du logiciel R, version 4.0.2 


\subsection{Discussion}

Les résultats du tableau $\mathrm{n}^{\circ} 4$ montrent l'influence significative de certaines variables sur la performance sociale, financière et globale des IMF en Côte d'Ivoire, de la période 2013-2018.

La rentabilité des actifs (ROA) a un effet positif sur la contre-performance sociale des IMF. Elle est significative à $5 \%$. En d'autres termes, la rentabilité des actifs « ROA» influence négativement la performance sociale. Ce résultat pourrait traduire que les dirigeants des IMF exercent une mauvaise gestion de leurs actifs et donc une performance financière moindre. En effet, la gestion perfectible des actifs entrainerait des charges élevées, ce qui pourrait contribuer moins efficacement à l'amélioration du bien-être et à la réduction de la pauvreté. Ce résultat ne répond pas à notre attente.

Outre ce résultat, la variable «formel », représentant les IMF de type coopératif est significative à $10 \%$. Elle influence positivement l'inefficacité sociale. En d'autres termes, les IMF de types coopératifs ont un effet négatif sur la performance sociale, c'est-à-dire ne sont pas performantes socialement. Cela signifie que les mutuelles ou coopératives contribuent moins au financement des projets à des personnes pauvres ou participent moins au bien-être de ces personnes. Ce résultat corrobore la thèse des institutionnalistes qui montre que le ciblage des populations très pauvres est coûteux tout comme les opérations de prêt à cette clientèle, notamment les coûts liés à l'octroi d'un grand nombre de plus petits prêts. Il ne répond pas à notre attente. Nos résultats sont contraires à ceux d'Adair et Berguiga (2010) qui montrent que les coopératives ou mutuelles, au regard de leur statut, peuvent avoir la capacité d'atteindre les plus pauvres.

Les estimations révèlent aussi que les IMF de petites tailles «taille1 », dont les encours de crédit sont inférieurs à 1 milliard Fcfa ne sont pas performantes financièrement. Ces petites IMF exercent soit une gestion perfectible de leurs ressources ou ne disposent pas de qualifications nécessaires pour une bonne gestion de l'IMF. Cette situation peut occasionner des charges supplémentaires et entrainer en moyen termes la faillite de l'institution. Nos résultats ne corroborent pas ceux de Bassem (2008) qui trouve que, les institutions de microfinance de tailles moyennes présentent une plus grande efficacité technique. Les résultats sont également différents de ceux de Kablan (2012), Akhavein (1997) et Smirlock (1985) qui trouvent une relation positive et significative entre la taille et la rentabilité des banques (op. cit. Benzai).

Enfin, nous montrons que les IMF nouvelles «agel» ont une influence négative sur la performance globale. Ceci traduit que les IMF nouvelles n'ont pas encore atteint la phase de maturité pour être performantes socialement et financièrement. Car, plus une IMF murit, elle acquiert de l'expérience, mieux elle parvient à gérer ses coûts et à mettre en place de meilleurs mécanismes de gestion du risque et mieux, elle atteindra ses objectifs, et par la suite, elle est plus performante socialement et financièrement. Nos résultats corroborent ceux de Tobga (2019) qui indiquent que, le fait 
qu'une institution de microfinance soit nouvelle (âge compris entre 1 et 5 ans), l'amènerait à être moins performante en matière de pérennité financière.

\section{Conclusion}

Sur la période 2013-2018, la microfinance en Côte d'Ivoire a connu une évolution contrastée. Cependant, les perspectives restent favorables pour le secteur, au regard de l'accroissement du nombre de clientèle, de la restructuration de l'UNACOOPEC-CI ainsi que de la poursuite des actions d'assainissement. Notre étude a permis d'identifier les facteurs susceptibles d'influencer les performances sociale, financière et globale des IMF en Côte d'Ivoire. Les résultats de nos estimations permettent de conclure que la rentabilité des actifs ( $R O A)$, les IMF de type coopératif, la taille des IMF et les IMF nouvelles (âge) ont une influence significative sur la performance de ces institutions. Ainsi, il serait souhaitable que les IMF de petites tailles réduisent le volume de leurs crédits pour leur permettre d'adopter une bonne gestion de leurs structures et être performantes. Ensuite, la formation des dirigeants et des responsables de crédit est nécessaire pour l'efficacité de leur institution. En outre, les dirigeants des IMF «jeunes » devraient organiser des formations pour le personnel afin d'être compétitives et performantes. Ils peuvent aussi s'inspirer des expériences des IMF performantes dans le secteur de la microfinance. Enfin, les pouvoirs publics doivent accentuer les efforts en termes de respect de la réglementation et de la formation ou de recyclage, de façon régulière pour permettre aux IMF de se conformer aux textes et de disposer de meilleurs outils de gestion.

Nous espérons que ce travail puisse constituer un outil de sensibilisation et de prise de conscience des acteurs de la microfinance en Côte d'Ivoire afin de permettre au secteur d'être performante socialement et financièrement.

\section{BIBLIOGRAPHIE}

[1] Adair, P. \& Berguiga, I. "Les facteurs déterminants de la performance sociale et de la performance financière des institutions de microfinance dans la région mena : Une analyse en coupe instantanée". Région et Développement, $n^{\circ} 32$, 2010, 91-120. http://econpapers.repec.org

[2] Akella, S. R., \& Greenbaum, S. I. “A note on savings and Loans Ownership Structure and expense preference: A reexamination". Journal of Banking and Finance, vol.12, $n^{\circ} 3$, 1988, 419-437. https://doi: 10.1016/S0378-4266(02)003151

[3] Balkenhol B. "Microfinance and Public Policy: Outreach, Performance and efficiency", Palgrave Macmillan, 2007.

[4] Bassem, B.S. "Efficiency of Microfinance Institutions in the Mediterranean: An Application of DEA”. Journal Transition Studies Review, Vol.15 (2). 2008, 343-354. https://doi: 10.1007/s11300-008-0012-7

[5] Battilana J. \& Dorado, S. "Building Sustainable Hybrid Organizations: The case of Commercial Microfinance Organizations". Academy of Management Journal, vol.53, (6), 2010, 1419-1440. https://doi: 10.5465/AMJ.2010.57318391

[6] Benzai Y. "Mesure de l'Efficience des Banques Commerciales Algériennes par les Méthodes Paramétriques et Non Paramétriques". (Thèse de Doctorat, en Sciences Economiques, Ecole doctorale : Management International Des Entreprises, Université Abou Bakr Belkaid -Tlemcen), 2016, http://dspace.univ-tlemcen.dz/handle/112/8724

[7] Benston, G.J. “'Branch banking and economies of scales”. The Journal of finance, vol.20. (2), 1965, 312-331. https://doi: 10.1111/j.1540- 6261.1965.tb00212.x 
[8] Berg S., Forsund F., Hjalmarsson L., Suominen M. "Banking efficiency in the nordiv Countries”. Journal of Banking and Finance 17, 1992, 371-388.

[9] Betl, F.W. and Murphy, N.B. "Costs in commercial banking: a quantitative analysi of bank behaviour and its relation to bank regulation”. Report, Boston: Federal Reserve Bank of Boston, $\mathrm{n}^{\circ} 41,1968$.

[10] Brown, J. "Developing a movement through community development and microfinance: a case study of the Federation of Homeless People in Zimbabwe". WorldCat.Org Thesis, University of Toronto, Ontario, Canada. 2005, http://www.worldcat.org/

[11] Caudill, S.B., Gropper et V. Hartarska D.M. "Which Microfinance Institutions Are Becoming More Cost Effective with Time? Evidence from a Mixture Model". Journal of Money, Credit and Banking (JMCB), vol.41(4), 2009, 651-672. https://doi: $10.1111 / \mathrm{j} .1538-4616.2009 .00226 . \mathrm{x}$

[12] Charnes, A., Cooper, W. W., \& Rhodes, E. “Measuring the Efficiency of Decision Making Units”. European Journal of Operational Research, vol.2, ${ }^{\circ}{ }^{\circ}$, 1978, 429-444. https://doi: 10.1016/0377-2217(78)90138-8

[13] Commission Nationale pour la microfinance. "Rapport annuel 2010 de la Commission Nationale pour la microfinanceCôte d'Ivoire", 2010

[14] Coelli, T. J., Rao, D.S.P., O'Donnell, C.J., Battese, G.E. “An Introduction to Efficiency and Productivity Analysis". Kluwer Academic Publishers, Boston, 331p. $2^{\text {nd }}$ ed., 1998, https://doi : $\underline{10.1007 / 978-1-4615-5493-6}$

[15] Cornée, S., \& Thenet, G. "Efficience des institutions de microfinance en Bolivie et au Pérou : une approche Data Envelopment Analysis en deux étapes". Finance Contrôle Stratégie, Association FCS, 2016, vol. (19-1)/2016, 〈halshs013069> https://doi : 10.4000/fcs.1768

[16] De Briey V. “Pleins feux sur la microfinance en 2005”. Regards Economique n²8, IRES-UCL, Louvain- La-Neuve, 2005

[17] Direction Générale du Trésor et de la Comptabilité Publique \& Direction de la réglementation et de la surveillance des systèmes financiers décentralisés. "Monographie des Systèmes financiers décentralisés, Côte d'Ivoire-2018”, version 1, 2018.

[18] Djimasra, N. “Efficacité technique, productivité et compétitivité des principaux Pays producteurs de coton”. Thèse de doctorat en sciences économiques, Économies et finances. Université D’ORLEANS, 2009, HAL Id: <NNT: 2009ORLE0507 >.429p. https://tel.archives-ouvertes.fr

[19] Djontu M. A. "Problématique de la performance des institutions de microfinance Camerounaises: Une analyse des déterminants". Revue Internationale des Sciences de Gestion Vol. 2 (3), 2019, 23-46

[20] Fama E. F. 'Stock returns, expected returns and real activity”. Journal of financial economics, XLV, 4 sept, 1990, 10891108.

[21] Gonzalez, A. "Efficiency Drivers of Microfinance Institutions (MFIs): The Case of Operating Costs". Microbanking Bulletin, 15, 2007, 37- 42.

[22] Gutiérrez-Nieto, B., Serrano-Cinca C. \& Mar-Molinero C. "Social Efficiency in Microfinance Institutions". The Journal of the Operational Research Society, vol.60, (1), 2009, 104-119. https://doi:10.1057/palgrave.jors.2602527

[23] Gutiérrez-Nieto B., C. Serrano-Cinca et C. "Mar-Molinero. Microfinance Institutions and efficiency". The International Journal of Management Science, vol.35. (2), 2007, 131-142. https://doi:10.1016/j.omega.2005.04.001

[24] Haq, M., Skully, M. \& Pathan S. "Efficiency of Microfinance Institutions: A Data Envelopment Analysis”. Journal of Asia-Pacific Financial Markets, vol.17 (1), 2010, 63-97. https://doi: 10.1007/s10690-009-9103-7

[25] Hansmann, H. “The Ownership of Enterprise. Harvard University Press". 384p, 1996

[26] Hartarska V. \& Mersland R. "Which Governance Mechanisms Promote Efficiency In Reaching Poor Clients? Evidence from Rated Microfinance Institutions". European Financial Management, vol.18 (2), 2012, 218-239. https://doi : 10.1111/j.1468-036X.2009.00524.X

[27] Hassan K.M. et Sanchez B. "Efficiency Analysis of Microfinance Institutions in Developing Countries”. Cahier de recherché n ${ }^{\circ}$ 2009-WP-12 du Networks Financial Institute. 2009, https://doi: 10.2139/ssrn.1492238

[28] Hermes, N., Lensink, R. \& Meester, A. “Outreach and Efficiency in Microfinance Institutions". World Development, vol. 39, (6), 2011, 938-948. https://doi: org/10.1016/j.worlddev.2009.10.018

[29] Hudon M. \& Balkenhol B. “Efficiency. In B. Armendariz et M. Labie (éds.)”,The Handbook of Microfinance. LondonSingapore: World Scientific Publishing, 2011, 383-396p, http://www.worldscientific.com/

[30] Hulme, D., Moore, K. “Why Has Microfinance Been a Policy Success in Bangladesh?” in Bebbington, A., McCourt, W. (dir.), Development Success: Statecraft in the South, Palgrave Macmillan, Basingstoke. 2007 
[31] Institut national de la statistique \& Direction générale du plan et de la lutte contre la Pauvreté. “Enquête sur le niveau de vie des ménages en côte d'Ivoire (ENV 2015)", (2015, juillet)

[32] Kablan, "Efficacité des institutions de microfinance en UEMOA : une approche outreach - intermédiation financière". Equipe de Recherche sur l'Utilisation des Données Individuelles Temporelles en Economie (ERUDITE); 2012, Université Paris-Est Créteil Val-de-Marne (UPEC), https://halshs.archives-ouvertes.fr/

[33] Kendo C. "La consolidation en microfinance : Le cas africain. Thèse de doctorat en sciences économiques". 2014, Université de Lorraine. http://www.theses.fr/

[34] Kobou G., Ngoa H. et Moungou S., "L'efficacité du financement des micro et petites entreprises dans la lutte contre la pauvreté au Cameroun". Economie Appliquée, vol.63 (1), 2010, 135-162.

[35] Lafourcade, A-L., Isern, J., Mwangi, P. \& Brown, M. "Étude sur la portée et les performances financières des institutions de microfinance en Afrique". The Micro Banking Bulletin, vol.12, 2005, 3-21. http://www.doc-developpementdurable.org/file/

[36] Mersland, R. \& Strøm, R. Ø. “Performance and Governance in Microfinance Institutions”. Journal of Banking \& Finance, vol.33(4),2009, 662-669. https://doi: 10.1016/j.jbankfin.2008.11.009

[37] Morduch J. "The microfinance schism". World Development, vol.28 (4), 2000, 617-629. https://doi: $\underline{10.1016 / \text { S0305- }}$ 750X (99)00151-5

[38] Morduch, J. "The Microfinance Promise”, The Journal of Economic Literature, 67, 1999a, 1569-1614.

[39] Morvant-Roux S. "Is microfinance the adequate tool to finance agriculture? In Armendariz B, \& M. Labie (Eds.), Handbook of microfinance". Washington, DC: World Scientific Publishing, 2011, https://www.unifr.ch/heed/assets/.pdf

[40] Myers S. C. et Majluf N. S. "Corporate financing and Investment decisions when firms have information that investors do not have". Journal of Financial Economics, 13, 1984, 187-221.

[41] N'gbo, A. G. M. “Frontière de production et efficacité : Une analyse des scop françaises". Thèse de Doctorat en sciences économiques, 1991, Université des Sciences Sociales de Toulouse. http://greenstone.lecames.org/

[42] Nghiem, H.S, Coelli T. et Rao, P. "The Efficiency of Microfinance in Vietnam: Evidence from NGO Schemes in the North and the Central Regions". International Journal of Environmental, Cultural, Economic and Social Sustainability, vol.2, (5), 2006, 71-78, http://espace.library.uq.edu.

[43] Nzongang, J. "La mesure de la performance des établissements de microfinance (EMF) au Cameroun une application combinée DEA et multicritère au cas du réseau des Mutuelles Communautaires de Croissance (MC2)". Revue des Sciences de Gestion, Vol.3, (249-250), 2011, 139-146. https://doi : 10.3917/rsg.249.0139

[44] Ndjokou M.M. "Ciblage des Pauvres et Rentabilité Financière dans les Institutions de Microfinance au Cameroun: Complémentarité Ou Incompatibilité?" Investment Climate and Business Environment Research Fund, Rapport de recherche du FR-CIEA N0. 53/13, 30p, 2013, http://trustafrica.org/

[45] Nzongang J. Piot-Lepetit I. Kamdem D. "La mesure de l'efficacité financière et sociale des institutions de microfinance $\mathrm{du}$ réseau $\mathrm{MC}^{2}$ au Cameroun»". Mondes en développement, vol.4, (160), 2012, 99-116. https://doi: 10.3917/med.160.0099

[46] Périlleux A. "Strategic Governance Lessons from History for West African Microfinance Cooperatives". Journal of Strategic Change, vol.22, (1-2), 2013, 95-106. https://doi: 10.1002/jsc.1924

[47] Qayyum, A., Ahmad, M., "Efficiency and Sustainability of Microfinance". Munich Personal RePEc Archive-MPRAWorking Paper, $\mathrm{n}^{\circ} 11674$, University Library of Munich, 2006, 1-38. https://mpra.ub.uni-muenchen.de/11674/

[48] Sealey, C., \& Lindley, J. "Inputs, outputs, and a theory of production and - cost at depository financial institutions". Journal of Finance, vol.32,(4), 1977, 1251-1266. http://links.jstor.org/sici?

[49] Servin, R., Lensink, R. \& Van den Berg, M. "Ownership and Technical Efficiency of Microfinance Institutions: Empirical Evidence from Latin America”. Journal of Banking \& Finance, vol.36, (7), 2012, 2136-2144. https://doi: 10.1016/j.jbankfin.2012.03.018

[50] Sika, G.L. “Impact des allocations en ressources sur l'efficacité des écoles primaires en Côte d'Ivoire”. Economies et finances, Université de Bourgogne, Thèse de doctorat en sciences économiques, Université de Bourgogne, 2011, https://hal.inria.fr/file/index/docid/.pdf

[51] Simar, L. \& Wilson, P.W. "Estimation and Inference in Two-Stage, Semi- Parametric Models of Production Processes". Journal of econometrics, vol. 136, (1), 2007, 31-64. https://doi : 10.1016/j.jeconom.2005.07.009 
[52] Solhi, S. \& Rigard, M. "Pérennité et efficience des institutions de microfinance dans la région mena". Economic Research Forum-Working Paper series, $\mathrm{n}^{\circ} 829 . \quad$ Université Mohammed V- Agdal-Rabat, Maroc. 29p, 2014. http://docplayer.fr/1007331

[53] Soulama, S. "Efficacité technique et inefficience à l'échelle des institutions de microfinance au Burkina Faso". Laboratoire d'Economie d'Orléans, 24p, 2008.https://www.microfinancegateway.org

[54] Sufian, F. "The efficiency of Islamic banking industry: a non-parametric analysis with non-discretionary input variable". Islamic Economic Studies Vol.14, (1 \& 2), 38, 2006. http://www.irti.org/.pdf

[55] Thenet G. "Une validation des temps standards d'opération dans le secteur des services”. Comptabilité, Contrôle, Audit et Institution(s), Tunisie. pp. CD-Rom, 2006. 〈halshs-00548049>

[56] Togba E.L.P. “Performance de la microfinance en Afrique de l'Ouest”. Série de documents de travail $N^{\circ} 319$, Banque africaine de développement, Abidjan, Côte d'Ivoire, 2019.

[57] Varman M. "Benchmarking microfinance institutions in India and determinants of their technical efficiency". Indian Journal of Economics and Business, Vol.7, (2), 2008, 255-265.

[58] Vanroose, A. “What Macro Factors Make Microfinance Institutions Reach Out?” Savings and Development, 32, 2008, 153-174.

[59] Wijesiri, M., Viganò L. \& Meoli M. "Efficiency of Microfinance Institutions in Sri Lanka: A Two-Stage Double Bootstrap DEA Approach". Economic Modelling, vol.47, 2015, 74-83. https://doi: 10.1016/j.econmod.2015.02.016

[60] Xue, M. et Harker, P.T. ' Overcoming the Inherent Dependency of DEA Efficiency Scores: A Bootstrap Approach”. Working paper 99-17, Wharton Financial Institution Center, University of Pennsylvan, 1999

[61] Yaron J. “Assessing Development Finance Institutions: A Public Interest Analysis”, World Bank Discussion Paper, 340 , Washington D.C, 1997. 